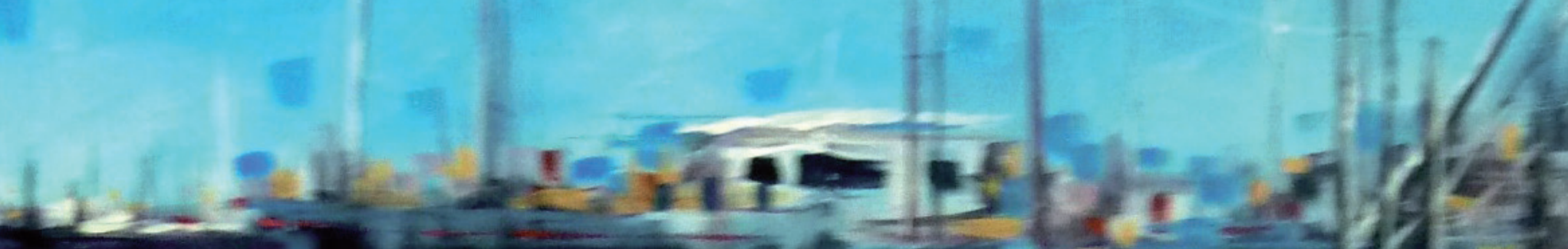




\section{La mortalidad infantil en Argentina. Análisis de sus cambios y de las diferencias regionales.}

Victoria MAZZEO*

RESUMEN. El objetivo de este artículo, es analizar, a partir de mediados del siglo XX, los cambios en el nivel de la mortalidad infantil de Argentina observando las diferencias regionales. Una primera lectura indica que en Argentina, entre los años 1950 y 2010, el nivel de la mortalidad infantil descendió más del 80\%, siendo su nivel de 11,1 por mil en 2012. Al tener en cuenta el comportamiento de las distintas jurisdicciones se advierte que sólo una tercera parte de ellas se mantuvieron durante el siglo XXI por debajo del promedio del país y otra tercera parte estuvo por encima, el resto presentó altibajos.

Palabras clave: mortalidad infantil - neonatal - postneonatal - causas reducibles.

SUMMARY. The objective is to analyze, from the mid-twentieth century, changes in the level of infant mortality in Argentina watching, regional differences. A first reading indicates that in Argentina, between 1950 and 2010, the level of infant mortality declined more than $80 \%$, being its level of 11.1 per thousand in 2012 . Considering the behaviour of the various jurisdictions, only a third of them remained in the XXI century below the national average and a third part over, the rest presented ups and downs.

Keywords: infant mortality - neonatal - postneonatal - preventable causes.

\section{Introducción}

Disfrutar de buenas condiciones de salud es un derecho fundamental, cuyo logro, debe ser un objetivo prioritario de las políticas públicas. En el caso de los menores de un año, los principios de universalidad, interdependencia e indivisibilidad de los derechos, así como el principio del interés superior del niño, contenidos en la Convención sobre los Derechos del Niño de 1989, significó un cambio de paradigma respecto del concepto de infancia. A partir de allí, se los reconoce como sujetos plenos de derecho y se los considera como personas con necesidad de cuidados especiales. Al mismo tiempo, se otorgó un papel esencial a la familia en cuanto a la crianza, siendo reconocida como el lugar propio de convivencia y pertenencia de los niños. Se introdujo el nuevo paradigma de "protección integral", en el que la infancia es considerada una sola y su protección se expresa en la exigencia de formulación de políticas básicas universales para todos los niños (Mazzeo, 2006 y 2007).

La mortalidad infantil ha sido considerada como uno de los indicadores más sensibles de las condiciones de salud de la población, bajo el supuesto de que el niño al nacer está expuesto a ciertos factores del medio que inciden en su salud y que se reflejan en el nivel de la tasa de mortalidad infantil. Esos factores (ambiente sanitario, nutrición de la madre y del niño, programas de inmunización y control de enfermedades) se relacionan con el significado que tiene para un gobierno el concepto de bienestar social y con las políticas gubernamentales que lleva a cabo con ese fin. Por lo tanto, el estudio de la mortalidad infantil merece un lugar destacado entre los indicadores relativos al grado de desarrollo socioeconómico de una determinada sociedad. Esto explica por

Recibido: febrero de 2014 /Aceptado y versión final marzo de 2014.

* Jefa Departamento de Análisis Demográfico (dgesyc-gcba), Profesora Titular Regular de la Maestría Demografía Social (fsoc-uba) e Investigadora del Instituto Gino Germani (fsoc-uba), mail: victoria.mazzeo@gmail.com 
qué en los discursos de la dirigencia política se alude con frecuencia a esta variable y los responsables del sector salud se muestran muy sensibles a los cambios registrados en su nivel (Mazzeo, 2006).

Desde hace varios años, pero especialmente durante las tres últimas décadas, estudios teóricos y empíricos demostraron significativas diferencias en las condiciones de salud entre diferentes grupos sociales definidos de acuerdo con su nivel de ingresos, educación, origen étnico, género, área de residencia o factores ambientales. En América Latina, varios estudios confirmaron la existencia de una elevada asociación entre las probabilidades de sobrevivencia de los niños y los niveles socioeconómicos de los hogares (Breilh y Granda, 1983; Ruzicka, 1983; Bronfman y Tuirán, 1983; Mosley y Chen, 1984; Laurell, 1986; Puffer y Serrano, 1988; Behm, 1992; Menéndez, 1992; Moreno, 1999; Vinocur, 1999; CEPAL, 2000; Delamonica et. al., 2000; Mc Question, 2001; Mazzeo y Pollero, 2005).

En Argentina, varios estudios confirmaron la existencia de una elevada asociación entre las probabilidades de sobrevivencia de los niños y los niveles de pobreza de sus hogares (INDEC, 1984; Torrado, 1986 y 1992). También se investigaron las relaciones entre infancia y condiciones de vida (López y Tamargo, 1995 y Cid, 1995), observando que el riesgo de muerte y el deterioro en su crecimiento era sensiblemente más elevado entre los niños nacidos en hogares de pobreza estructural. Otros trabajos relacionaron el estado nutricional con el nivel económico-social de los hogares (Calvo, 1995 y Ministerio de Salud, 1999), todos ellos confirmaron una fuerte asociación entre prevalencia de desnutrición y pobreza. Se afirmó que el déficit de talla es reflejo de un proceso crónico en el que confluyen varios factores, particularmente el bajo peso al nacer, la carencia marginal de energía y nutrientes, la reiteración de procesos infecciosos agudos y las condiciones desfavorables del medio en que vive el niño.
A principios de este siglo, se encuentran los trabajos que analizan los diferenciales de la mortalidad infantil por tamaño de las localidades. Aportes a esta línea de investigación (UNICEF-INDEC, 2003; Celton y Ribotta, 2004; Ceballos, 2005; Trifiró, 2007; Mazzeo, 2008) muestran las diferencias en la sobrevivencia cuando se consideran las condiciones de la vivienda, el saneamiento ambiental y el nivel educativo de las madres, variables muy relacionadas con las condiciones socioeconómicas de los hogares. Se concluye que, independientemente de la región de residencia, son las condiciones socioeconómicas las que tienen mayor incidencia en la tasa de mortalidad de la infancia. En otra línea de investigación, se verificó el cumplimiento de las metas del año 2000 para la mortalidad infantil en Argentina (Mazzeo, 2003).

En síntesis, todos los estudios enfatizan la existencia de sistemáticas diferencias entre los grupos sociales, cualquiera sea el indicador que se utilice para clasificar estos grupos. Aquellos donde el riesgo es mayor son siempre los conglomerados sociales de más bajo nivel socioeconómico.

\section{Objetivo y metodología}

El objetivo es investigar, a partir de mediados del siglo $\mathrm{XX}$, los cambios en el nivel de la mortalidad infantil de Argentina, observando su composición por grupo etario (neonatal y postneonatal ${ }^{1}$ ) y los diferentes comportamientos regionales. Además, a partir de los 80 , es analizar la participación de las causas de muerte y en el período 2000-2012, comparar las diferencias jurisdiccionales.

Para la medición del nivel de la mortalidad infantil, se utilizó la tasa definida como la relación entre las defunciones de niños menores de un año, en un año dado y los nacidos vivos en el año ${ }^{2}$ Los datos provienen de las estadísticas vitales del período. En Argentina, los dos hechos vitales que intervienen en esta tasa (nacido vivo y defunción) son de inscripción obligatoria y las

1. Las muertes neonatales transcurren desde el nacimiento hasta los 27 días cumplidos y las postneonatales desde los 28 días hasta los 11 meses cumplidos. 2. Se discriminan en el análisis los grupos etarios que abarca la mortalidad infantil (neonatal y postneonatal). 
definiciones conceptuales son las recomendadas por la Organización Mundial de la Salud.

Se ha demostrado que el nivel de la mortalidad está relacionado con la evolución de la estructura de las causas de muerte. Para efecto de observar este proceso se consideraron ocho grupos de causas, a saber: ciertas afecciones originadas en el período perinatal (complicaciones del embarazo, trabajo de parto y del parto, trastornos relacionados con la duración corta de la gestación y el crecimiento fetal lento, etc.); anomalías congénitas (hidrocefalia, espina bífida, vicio orgánico al corazón y otras malformaciones congénitas); infecciones respiratorias agudas (neumonía, influenza, bronquitis y bronquiolitis agudas y las demás infecciones respiratorias agudas); enfermedades infecciosas intestinales (infecciones intestinales debidas a virus y otros organismos especificados, diarrea y gastroenteritis de presunto origen infeccioso, etc.); enfermedades del corazón (insuficiencia cardíaca, enfermedades isquémicas del corazón, etc.); deficiencias de la nutrición (desnutrición); accidentes (de transporte y otras traumatismos accidentales) y resto de las causas bien definidas. Además, para el último año del período en estudio, se analiza la mortalidad según criterios de reducibilidad ${ }^{3}$.

El estudio de las desigualdades regionales, se realizó a partir del agrupamiento de las 24 jurisdicciones que componen el país, en seis regiones. La primera está integrada por la Ciudad de Buenos Aires (capital del país) y el resto son: Pampeana (Buenos Aires, Córdoba, Entre Ríos y Santa Fe); Cuyo (Mendoza, San Juan y San Luis); Noreste (Corrientes, Chaco, Formosa y Misiones); Noroeste (Catamarca, Jujuy, La Rioja, Salta, Santiago del Estero y Tucumán) y Patagónica (Chubut, La Pampa, Neuquén, Río Negro, Santa Cruz y Tierra del Fuego).

Finalmente, con el objeto de mostrar cómo el promedio nacional ha evolucionado en sus componentes jurisdiccionales a lo largo del siglo XXI, se examina, para el período 2000/2012, la distribución de la mortalidad infantil en el país. Con ello se busca poner de relieve las desigualdades que en materia de mortalidad infantil se dan en el territorio nacional, y mostrar la necesidad de prestar atención focalizada hacia regiones y sectores de la población postergados en el acceso a servicios básicos de salud. A tal efecto, se han agrupado los comportamientos de la mortalidad infantil según división política territorial en cinco categorías: provincias que durante todo el período estuvieron por encima del promedio de país; las que estuvieron por debajo; las que comenzaron el período por encima y luego estuvieron por debajo; las que comenzaron por debajo y luego estuvieron por encima y las provincias con altibajos.

\section{La inserción de la mortalidad infantil de Argentina en el contexto internacional}

Con el objeto de conocer la inserción de la mortalidad infantil de Argentina en el contexto internacional, se comparó su nivel con las cifras de la estadística internacional de los países europeos y latinoamericanos.

En el Cuadro 1 se observa que desde mediados del siglo XX se produce un descenso sostenido de la mortalidad infantil en la mayoría de los países europeos. Por su

Cuadro 1. Tasa de mortalidad infantil en países europeos seleccionados y en Argentina, años 1950-2010.

\begin{tabular}{|l|c|c|c|c|c|c|c|}
\hline Países & 1950 & 1960 & 1970 & 1980 & 1990 & 2000 & 2010 \\
\hline Alemania $^{a}$ & 56 & 34 & 24 & 13 & 7 & 5 & 3 \\
\hline Argentina & 68 & 62 & 61 & 33 & 26 & 17 & 12 \\
\hline Austria & 66 & 38 & 26 & 14 & 8 & 5 & 4 \\
\hline Bélgica & 53 & 31 & 21 & 11 & 8 & 5 & 4 \\
\hline España & 70 & 46 & 28 & 12 & 8 & 4 & 4 \\
\hline Francia & 52 & 27 & 15 & 10 & 8 & 4 & 3 \\
\hline Hungría & - & 48 & 36 & 23 & 15 & 8 & 5 \\
\hline Italia & 64 & 44 & 29 & 14 & 9 & 5 & 3 \\
\hline Portugal & 94 & 78 & 58 & 26 & 13 & 6 & 3 \\
\hline Suecia & 21 & 17 & 15 & 7 & 6 & 3 & 2 \\
\hline
\end{tabular}

Nota: a Las tasas presentadas corresponden a la República Federal de Alemania y a partir de 1990 nuevamente a Alemania.

Fuente: elaboración propia en base a Mazzeo, 2006 y UNICEF, 2012.

3. El Sistema Estadístico de Salud de Argentina utiliza la clasificación de causas de muerte según Criterios de Reducibilidad, basada en la clasificación según criterios de "evitabilidad" elaborada en Chile por la Dra. Erica Taucher y que con su asesoramiento, fue adaptada a las modalidades de atención de la salud en Argentina. Su última revisión es de 2011. (Ministerio de Salud, Presidencia de la Nación y Sociedad Argentina de Pediatría, 2012). 
parte, Argentina inició el período con valores similares a algunos de los países europeos seleccionados (Austria, España, Italia) e incluso menor (Portugal). Esta situación se modificó a partir de 1970, cuando comenzó a registrar niveles superiores a los países europeos seleccionados, y aumentó la brecha que la separaba del país de menor nivel: en 1970 era cuatro veces el nivel de Suecia y seis veces en 2010. Argentina, partiendo de un nivel alto en 1950 (tres veces superior a Suecia), se redujo el 82\%, mientras que Suecia lo hizo el 90\%. Es decir, a pesar de iniciar el período con un nivel bastante más alto, su reducción fue menor.

Un párrafo aparte merece la comparación con la mortalidad infantil de América Latina. Argentina, durante todo el período (Cuadro 2) se mantuvo en un nivel intermedio entre los países latinoamericanos. Aquí también aumentó la brecha respecto al nivel mínimo. En 1950-55, era 1,1 veces el nivel mínimo latinoamericano (Uruguay) y en el quinquenio 2005-2010 aumenta a 2,6 veces el nivel mínimo, que desde 1970-1975 pertenece a Cuba.

El descenso sostenido de la mortalidad infantil de Argentina cercano al $80 \%$ no fue suficiente, la hizo descender en el ranking latinoamericano del segundo

Cuadro 2. Tasa de mortalidad infantil (por mil) estimada según quinquenios. América Latina y Argentina, años 19502010.

\begin{tabular}{|l|c|c|c|c|}
\hline Quinquenio & $\begin{array}{c}\text { América } \\
\text { Latina }\end{array}$ & $\begin{array}{c}\text { Nivel } \\
\text { máximo }\end{array}$ & $\begin{array}{c}\text { Nivel } \\
\text { mínimo }\end{array}$ & Argentina \\
\hline $1950-1955$ & 127,7 & 219,6 & 57,5 & 65,9 \\
\hline $1955-1960$ & 114,2 & 193,5 & 53,0 & 60,4 \\
\hline $1960-1965$ & 102,0 & 170,5 & 47,9 & 59,7 \\
\hline $1965-1970$ & 92,1 & 157,5 & 47,1 & 57,4 \\
\hline $1970-1975$ & 81,4 & 151,3 & 38,5 & 48,1 \\
\hline $1975-1980$ & 69,6 & 131,2 & 22,3 & 39,1 \\
\hline $1980-1985$ & 57,3 & 122,1 & 17,4 & 32,2 \\
\hline $1985-1990$ & 47,3 & 100,1 & 15,9 & 27,1 \\
\hline $1990-1995$ & 38,7 & 85,5 & 15,3 & 24,4 \\
\hline $1995-2000$ & 31,9 & 70,3 & 9,6 & 21,8 \\
\hline $2000-2005$ & 25,4 & 56,3 & 6,1 & 15,0 \\
\hline $2005-2010$ & 21,7 & 48,8 & 5,1 & 13,4 \\
\hline V.R.\% 2010/1950 & $-83,0$ & $-77,8$ & $-91,1$ & $-79,7$ \\
\hline
\end{tabular}

Nota: nivel máximo: Haití (1950-1965 y 1980-2010) y Bolivia (1965-1980) y nivel mínimo: Uruguay (1950-1970) y Cuba (1970-2010).

Fuente:elaboración propia en base a CELADE, 2010. lugar que tenía en el quinquenio 1950-1955 (detrás de Uruguay) al quinto puesto, detrás de Cuba (5,1 por mil), Chile (7,2 por mil), Costa Rica (9,9 por mil) y Uruguay (13,1 por mil). Indudablemente, Cuba, Chile y Costa Rica, han sido los países que, en el concierto latinoamericano, aplicaron las políticas públicas más eficaces en la reducción de la mortalidad infantil.

\subsection{La estructura por edad en la determinación del} nivel de la mortalidad

Con la finalidad de explicar la tendencia seguida por la mortalidad infantil, se consideró necesario examinar su composición por edad. La mortalidad infantil abarca dos etapas: la neonatal que ocurre durante las primeras cuatro semanas y la postneonatal que se produce durante el resto del primer año de vida. Antes de abordar el análisis de los componentes de la mortalidad infantil conviene recordar que existe relación entre la edad del fallecido y la etiología del fallecimiento, pues la mayoría de las muertes por causa endógena (inmadurez del recién nacido, malformación y debilidad congénita y traumatismos del parto) se producen en el período neonatal y casi la totalidad de los fallecimientos postneonatales se deben a causas exógenas (enfermedades infecciosas, desnutrición o alimentación inadecuada y accidentes). Esto se explica en relación con la naturaleza de las causas de muerte, cuya gran mayoría después del primer mes de vida, están relacionadas con el medio ambiente en el que se desarrolla el niño y cuyo control se logró con los avances de la sanidad y la medicina. En cambio, una parte considerable de las causas de muerte en el primer mes de vida tienen un origen endógeno y son debidas a factores congénitos durante la vida intrauterina y otros asociados al parto. Sobre éstas los progresos fueron más lentos y su reducción requirió mayores esfuerzos (Mazzeo, 2006).

Cuando se observa la evolución de la mortalidad infantil distinguiendo los grupos etarios que la componen (Cuadro 3), se detectan claramente dos períodos de similar amplitud, pero con diferente ritmo de descenso: 1950-1975 y 1975-2010. Durante los primeros veinticinco años la mortalidad infantil desciende el 35\%, la neonatal el 15\% 


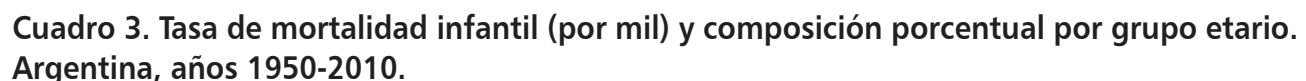
Argentina, años 1950-2010.

\begin{tabular}{|l|c|c|c|c|c|c|}
\hline Año & Total & Neonatal & Postneonatal & Total & Neonatal & Postneonatal \\
\hline 1950 & 68,2 & 26,0 & 42,2 & 100,0 & 38,1 & 61,9 \\
\hline 1955 & 61,8 & 24,8 & 36,9 & 100,0 & 40,1 & 59,7 \\
\hline 1960 & 62,4 & 26,6 & 35,8 & 100,0 & 42,6 & 57,4 \\
\hline 1965 & 56,9 & 24,8 & 32,1 & 100,0 & 43,6 & 56,4 \\
\hline 1970 & 61,2 & 25,5 & 35,7 & 100,0 & 41,7 & 58,3 \\
\hline 1975 & 44,4 & 22,0 & 22,4 & 100,0 & 49,5 & 50,5 \\
\hline 1980 & 33,2 & 18,7 & 14,5 & 100,0 & 56,3 & 43,7 \\
\hline 1985 & 26,2 & 16,4 & 9,8 & 100,0 & 62,6 & 37,4 \\
\hline 1990 & 25,6 & 15,6 & 9,4 & 100,0 & 60,9 & 36,7 \\
\hline 1995 & 22,2 & 13,6 & 8,1 & 100,0 & 61,3 & 36,5 \\
\hline 2000 & 16,6 & 10,9 & 5,7 & 100,0 & 65,7 & 34,3 \\
\hline 2005 & 13,3 & 8,8 & 4,5 & 100,0 & 65,9 & 33,7 \\
\hline 2010 & 11,9 & 7,9 & 4,0 & 100,0 & 66,7 & 33,8 \\
\hline
\end{tabular}

Fuente: elaboración propia en base a Mazzeo, 2001 y MSAS, 2013.

y la postneonatal el $47 \%$. En los segundos treinta y cinco años decrecen el 73\%, el 64\% y el 82\%, respectivamente. Evidentemente, el segundo período presenta un ritmo de descenso mayor: en el período 1950-1975 decrece en promedio el 1,4\% por año y en 1975-2010, el 2,1\% por año.

Como se muestra en el Gráfico 1, la evolución de la mortalidad infantil está fuertemente asociada al comportamiento del componente postneonatal. Es sabido que al disminuir el nivel de la mortalidad infantil adquiere importancia relativa la mortalidad neonatal. Esta última, en 1950 concentraba el 38\% de las muertes de menores de un año y aumenta al 67\% en 2010.

Gráfico 1. Tasa de mortalidad infantil (por mil) y estructura de sus componentes. Argentina, años 1950-2010

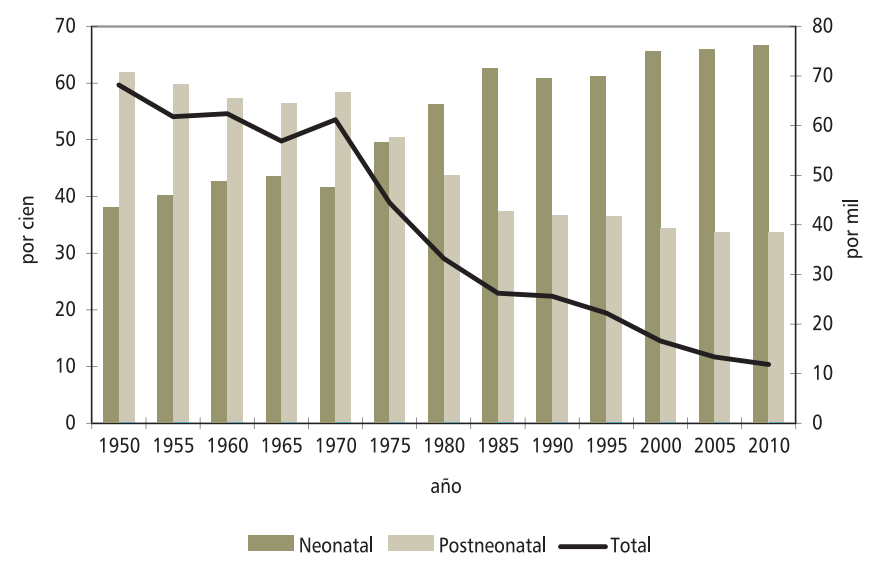

Fuente: elaboración propia en base a Mazzeo, 2001 y MSAS, 2013.
El ritmo de descenso de la mortalidad hacia el interior del país, fue muy heterogéneo (Cuadro 4). Las regiones de Cuyo, Noroeste y Patagónica, son las que descendieron más que el promedio del país $(82,6 \%)$, evidentemente partieron de niveles muy superiores $(82,1 ; 103,4$ y 97,6 por mil, respectivamente). La brecha entre el valor máximo y mínimo se redujo muy poco en el período presentado; si bien se destaca que se modificó la región que registraba el máximo. En 1950 la mortalidad infantil de la Región Noroeste era 2,8 veces la de Ciudad de Buenos Aires; mientras que en 2010 la Región Noreste registra 2,1 veces la de la ciudad.

En la Ciudad de Buenos Aires (durante todo el período registra el nivel menor), y en las Regiones Cuyo, Noroeste y Patagónica, el descenso de la mortalidad neonatal fue superior al promedio del país $(69,8 \%)$. En el caso de la mortalidad post-neonatal, como partieron de niveles más altos, fue mayor la caída en las Regiones Noroeste y Patagónica.

3.2 La participación de las causas de muerte en la determinación del nivel de la mortalidad

Existen evidencias de la relación entre el nivel de la mortalidad infantil por grupo etario y la evolución de la estructura de las causas de muerte. Por lo tanto, para una mejor comprensión del proceso del descenso 
Cuadro 4. Tasa de mortalidad infantil por grupo etario (por mil) según región. Argentina, años 1950-2010.

\begin{tabular}{|c|c|c|c|c|c|c|c|c|}
\hline \multirow[t]{2}{*}{ Región } & \multicolumn{8}{|c|}{ Tasa de mortalidad infantil } \\
\hline & 1950 & 1960 & 1970 & 1980 & 1990 & 2000 & 2010 & V.R. \% \\
\hline Total & 68,2 & 62,4 & 61,2 & 33,2 & 25,6 & 16,6 & 11,9 & $-82,6$ \\
\hline Ciudad de Buenos Aires & 37,5 & 40,4 & 31,5 & 18,5 & 16,8 & 9,4 & 7,0 & $-81,2$ \\
\hline Pampeana & 58,8 & 53,7 & 54,3 & 29,0 & 24,4 & 15,1 & 13,2 & $-77,5$ \\
\hline Cuyo & 82,1 & 66,2 & 63,6 & 31,9 & 23,1 & 16,0 & 11,4 & $-86,1$ \\
\hline Noreste & 71,4 & 69,4 & 75,4 & 48,8 & 33,1 & 24,3 & 15,2 & $-78,7$ \\
\hline Noroeste & 103,4 & 88,6 & 86,8 & 44,8 & 30,9 & 19,9 & 13,6 & $-86,8$ \\
\hline Patagónica & 97,6 & 97,4 & 77,8 & 33,5 & 20,8 & 14,8 & 9,2 & $-90,6$ \\
\hline \multirow[t]{2}{*}{ Región } & \multicolumn{8}{|c|}{ Tasa de mortalidad neonatal } \\
\hline & 1950 & 1960 & 1970 & 1980 & 1990 & 2000 & 2010 & V.R. \% \\
\hline Total & 26,0 & 24,5 & 25,5 & 18,7 & 15,6 & 10,9 & 7,9 & $-69,8$ \\
\hline Ciudad de Buenos Aires & 16,4 & 20,1 & 18,7 & 13,5 & 11,2 & 6,1 & 4,6 & $-71,9$ \\
\hline Pampeana & 22,1 & 22,0 & 23,9 & 17,9 & 15,4 & 9,7 & 8,6 & $-61,1$ \\
\hline Cuyo & 32,1 & 26,4 & 25,9 & 19,8 & 14,9 & 10,6 & 8,1 & $-74,7$ \\
\hline Noreste & 24,2 & 24,3 & 27,4 & 22,5 & 19,4 & 16,4 & 10,2 & $-57,7$ \\
\hline Noroeste & 39,8 & 32,3 & 32,0 & 20,2 & 16,8 & 13,3 & 9,1 & $-77,2$ \\
\hline Patagónica & 44,0 & 32,1 & 30,2 & 18,3 & 13,2 & 10,0 & 6,5 & $-85,3$ \\
\hline \multirow[t]{2}{*}{ Región } & \multicolumn{8}{|c|}{ Tasa de mortalidad post-neonatal } \\
\hline & 1950 & 1960 & 1970 & 1980 & 1990 & 2000 & 2010 & V.R. $\%$ \\
\hline Total & 42,2 & 37,9 & 35,7 & 14,5 & 9,4 & 5,7 & 4,0 & $-90,5$ \\
\hline Ciudad de Buenos Aires & 21,0 & 20,3 & 12,8 & 5,0 & 5,5 & 3,2 & 2,4 & $-88,4$ \\
\hline Pampeana & 36,7 & 31,7 & 30,4 & 11,1 & 8,6 & 5,4 & 4,6 & $-87,4$ \\
\hline Cuyo & 50,0 & 39,8 & 37,7 & 12,1 & 8,0 & 5,4 & 3,3 & $-93,5$ \\
\hline Noreste & 47,2 & 45,1 & 48,0 & 26,3 & 13,6 & 7,8 & 5,0 & $-89,5$ \\
\hline Noroeste & 63,7 & 56,3 & 54,9 & 24,6 & 12,5 & 6,5 & 4,5 & $-92,9$ \\
\hline Patagónica & 58,8 & 65,2 & 47,6 & 15,2 & 7,2 & 4,8 & 2,7 & $-95,4$ \\
\hline
\end{tabular}

Fuente: elaboración propia en base a Celton y Ribotta, 2004 y Ministerio de Salud, 2011.

de la mortalidad de los menores de un año, se consideró imprescindible efectuar el análisis de la mortalidad por causas. En general, el descenso de las muertes por causas exógenas (principalmente las enfermedades infecciosas y parasitarias y la diarrea) respondió al control de los factores del ambiente y también de la inmunización masiva de la población y al uso de nuevas drogas. De este modo, la estructura de la mortalidad por causas de muerte se modifica y cobran progresiva importancia otros grupos de causas, entre ellas, las de origen endógeno, es decir, las malformaciones congénitas y las originadas en el período perinatal (Mazzeo, 2006). Como se observa en el Cuadro 5, en Argentina en 1980, ya resulta evidente la menor proporción de muertes por causas de origen exógeno (in- fecciosas y parasitarias, diarrea y enteritis y respiratorias agudas) y la importancia de las muertes por causas de origen endógeno (anomalías congénitas y perinatales), llegando a representar estas últimas, a finales del período el 80\% de las muertes de menores de un año. Se destaca, el mayor aumento de las anomalías congénitas, que casi triplicó su participación.

Otra manera de abordar el análisis de la mortalidad infantil es a través de la clasificación de las causas de muerte según criterios de reducibilidad. Los criterios se refieren a la reducibilidad por distintas acciones, principalmente las desarrolladas en los servicios de salud. Se ha afirmado que la mayor incidencia de las muertes reducibles en el total de muertes neonatales y postneonatales 
Cuadro 5. Mortalidad infantil, composición porcentual de las causas de muerte. Argentina, años 1980 - 2010.

\begin{tabular}{|c|c|c|c|c|c|c|c|}
\hline Causas de muerte & 1980 & 1985 & 1990 & 1995 & 2000 & 2005 & 2010 \\
\hline Total & 100,0 & 100,0 & 100,0 & 100,0 & 100,0 & 100,0 & 100,0 \\
\hline Ciertas afecciones originadas en el período perinatal & 45,2 & 50,2 & 49,6 & 48,8 & 54,3 & 55,0 & 53,2 \\
\hline Anomalías congénitas & 10,3 & 13,0 & 14,6 & 17,9 & 22,5 & 24,6 & 27,0 \\
\hline Infecciones respiratorias agudas & 8,2 & 5,1 & 3,9 & 4,7 & 3,5 & 3,2 & 3,8 \\
\hline Enf. Infecciosas intestinales & 7,3 & 4,3 & 3,1 & 2,2 & 1,4 & 0,9 & 1,2 \\
\hline Enf. del corazón & 3,0 & 2,9 & 4,1 & 3,4 & 1,1 & 0,9 & 0,9 \\
\hline Deficiencias de la nutrición & 2,6 & 3,1 & 3,3 & 1,6 & 1,0 & 0,9 & 0,3 \\
\hline Accidentes & 2,7 & 2,8 & 3,8 & 3,9 & 4,4 & 3,4 & 2,6 \\
\hline Resto de las causas bien definidas & 20,7 & 18,6 & 17,6 & 17,5 & 11,6 & 11,0 & 11,1 \\
\hline
\end{tabular}

Fuente: elaboración propia en base a Mazzeo, 2001 y MSAS, 2002, 2007 y 2012.

es un indicador de la mala atención de la salud. Como ya se señalara, la última revisión de esta clasificación es de 2011, por lo tanto se presenta la composición de las muertes infantiles según estos criterios para el bienio 2011-2012.

$\mathrm{Al}$ analizar la participación de las muertes reducibles tanto en la mortalidad neonatal como postneonatal (Cuadro 6), se observa que las mismas representan una altísima proporción de muertes infantiles. En la mortalidad neonatal el $61 \%$ y en la mortalidad postneonatal el $67 \%$ de la muertes podrían evitarse.

Del análisis detallado de las causas de muerte reducibles se deduce que en la mortalidad neonatal prevalecen los factores relacionados preferentemente con el adecuado cuidado y tratamiento del recién nacido (enfermedades infecciosas intestinales, enfermedades bacterianas, anemias hemolíticas, tumores malignos, hidrocefalia, espina bífida, etc.) y en el período perinatal (VIH, trastornos relacionados con la duración de la gestación y el crecimiento fetal, etc.). Por su parte, en la mortalidad postneonatal tienen mayor impacto las reducibles mediante la implementación adecuada y oportuna del tratamiento correspondiente y la prevención (enfermedades infecciosas intestinales, enfermedades bacterianas, encefalitis, trastornos del metabolismo, etc.).

Si bien en las últimas décadas se ha comprobado la disminución de la mortalidad, pareciera que se ha producido un deterioro de ciertos indicadores de la salud infantil. Probablemente, el inestable contexto político e institucional haya imposibilitado la continuidad de algunos programas de atención de la salud materno-infantil y de esta manera, se haya frenando el descenso de la mortalidad infantil. Esto, a su vez, habría provocado la ampliación de las desigualdades sociales y espaciales en

Cuadro 6. Composición porcentual de la mortalidad infantil según grupo de edad y criterios de reducibilidad. Argentina, años 2011 y 2012.

\begin{tabular}{|c|c|c|}
\hline Mortalidad neonatal & 2011 & 2012 \\
\hline Total & 100,0 & 100,0 \\
\hline Reducibles & 61,2 & 61,0 \\
\hline Preferentemente en el embarazo & 2,3 & 2,7 \\
\hline Preferentemente en el parto & 0,8 & 0,7 \\
\hline Preferentemente en el recién nacido & 28,7 & 30,5 \\
\hline Por tratamiento clínico & 16,7 & 17,4 \\
\hline Por tratamiento quirúrgico & 1,8 & 1,5 \\
\hline Por tratamiento clínico y quirúrgico & 10,2 & 11,6 \\
\hline En el período perinatal & 28,8 & 26,4 \\
\hline Otras reducibles & 0,7 & 0,7 \\
\hline Difícilmente reducibles & 26,7 & 28,0 \\
\hline No clasificables & 9,4 & 9,3 \\
\hline Mal definidas e inespecíficas & 2,7 & 1,7 \\
\hline Mortalidad postneonatal & 2011 & 2012 \\
\hline Total & 100,0 & 100,0 \\
\hline Reducibles & 67,4 & 66,8 \\
\hline Por prevención & 0,2 & 0,4 \\
\hline Por tratamiento & 24,4 & 25,9 \\
\hline Por prevención y tratamiento & 36,1 & 33,1 \\
\hline Otras reducibles & 6,7 & 7,4 \\
\hline Difícilmente reducibles & 13,4 & 15,3 \\
\hline No clasificables & 8,0 & 7,7 \\
\hline Mal definidas e inespecíficas & 11,2 & 10,1 \\
\hline
\end{tabular}

Fuente:elaboración propia en base a MSAS, 2012 y 2013. 
la atención de la salud. Como se muestra en el Gráfico 2 , a fines del período analizado persisten altos niveles de mortalidad reducible.

Gráfico 2 Composición de las causas de mortalidad según criterios de reducibilidad en la mortalidad neonatal y postneonatal. Argentina, año 2012.
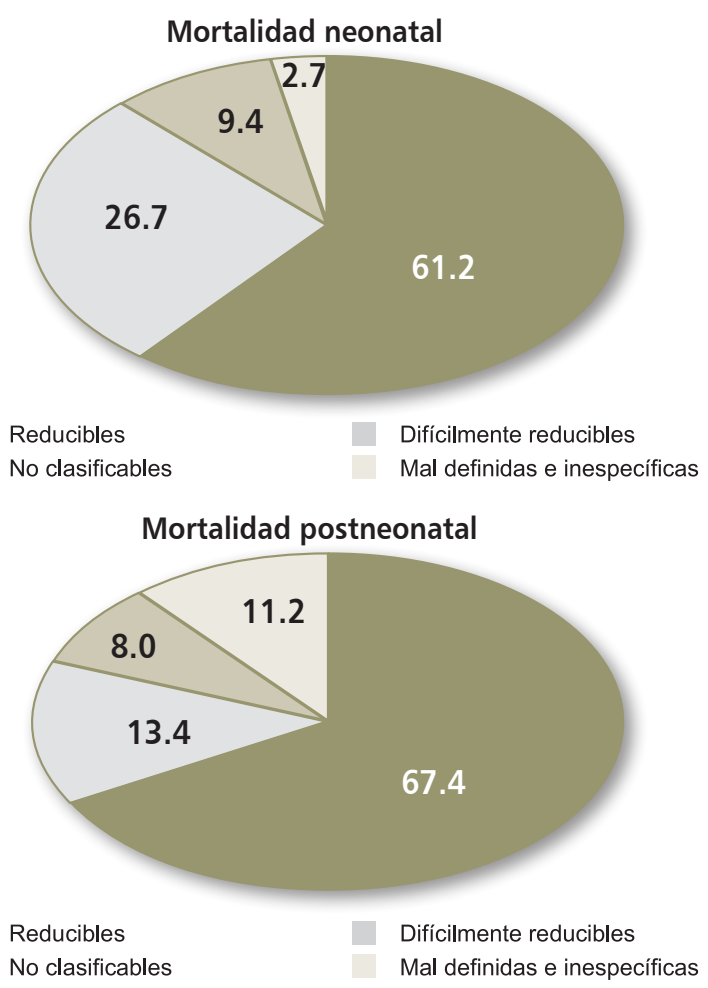

\subsection{La situación de la mortalidad infantil en el} siglo XXI

Durante el siglo XXI, al igual que en períodos anteriores, el nivel del componente post-neonatal determina la tendencia de la mortalidad infantil. Es decir, las reducciones más importantes de la mortalidad de menores de un año se deben a la disminución del componente post-neonatal (Cuadro 7). Entre los años 2000 y 2012, la mortalidad infantil desciende el 33\%, la neonatal el 31\% y la postneonatal el 36\%. La participación del componente neonatal se incrementa el 2,5\%. La reducción del nivel de la mortalidad neonatal es más difícil y requiere de acciones más complejas y costosas que la post-neonatal.

Si bien hasta aquí se ha analizado el comportamiento de la mortalidad infantil del total del país y por regiones, dicho comportamiento es diferencial en las jurisdicciones que lo componen. El propósito de considerar la distribución de la mortalidad infantil en el espacio, para el período 2000-2012, es mostrar cómo el promedio nacional ha evolucionado en sus componentes jurisdiccionales a lo largo del siglo XXI. Se busca poner de relieve las desigualdades que en materia de mortalidad infantil se dan en el territorio nacional, prestando atención a las jurisdicciones con mayores niveles.

Fuente: elaboración propia en base a MSAS, 2013.

Cuadro 7. Tasa de mortalidad infantil según grupo etario (por mil) y su composición porcentual. Argentina, años 2000/2012.

\begin{tabular}{|c|c|c|c|c|c|c|}
\hline Año & Total & Neonatal & Postneonatal & Total & Neonatal & Postneonatal \\
\hline 2000 & 16,6 & 10,9 & 5,7 & 100,0 & 65,7 \\
\hline 2001 & 16,3 & 10,6 & 5,6 & 100,0 & 65,2 \\
\hline 2002 & 16,8 & 11,3 & 5,6 & 100,0 & 66,9 & 34,5 \\
\hline 2003 & 16,5 & 10,5 & 5,9 & 100,0 & 63,9 & 33,1 \\
\hline 2004 & 14,4 & 9,7 & 4,6 & 100,0 & 67,8 & 36,1 \\
\hline 2005 & 13,3 & 8,9 & 4,5 & 100,0 & 66,3 & 32,2 \\
\hline 2006 & 12,9 & 8,5 & 4,4 & 100,0 & 65,7 & 34,7 \\
\hline 2007 & 13,3 & 8,5 & 4,8 & 100,0 & 64,1 & 35,9 \\
\hline 2008 & 12,5 & 8,3 & 4,2 & 100,0 & 66,3 & 33,7 \\
\hline 2009 & 12,1 & 8,0 & 4,1 & 100,0 & 66,0 \\
\hline 2010 & 11,9 & 7,9 & 4,0 & 100,0 & 66,3 \\
\hline 2011 & 11,7 & 7,6 & 4,1 & 100,0 & 64,8 \\
\hline 2012 & 11,1 & 7,5 & 3,6 & 100,0 & 67,3 \\
\hline V.R. $\%$ & $-32,9$ & $-31,2$ & $-36,3$ & V.R. $\%$ & 33,7 \\
\hline
\end{tabular}

Fuente:elaboración propia en base a MSAS, 2013. 
A tal efecto, como ya se señalara, se han agrupado los comportamientos de la mortalidad infantil según división política territorial en cinco categorías (Cuadro 8). Se observa que cerca de la mitad del país (42\%) se encuentra en condiciones desfavorables con respecto al promedio del país; allí se ubican las jurisdicciones que durante todo el período estuvieron por encima de dicho promedio (Catamarca, Corrientes, Chaco, Formosa, Jujuy, La Rioja, Misiones, Salta y Tucumán) y la que empeoró su situación, es decir comenzó el período por debajo del promedio del país y luego estuvo por encima (Buenos Aires).

Otro $42 \%$ concentran las provincias que muestran condiciones favorables. Están las que durante todo el período estuvieron por debajo del promedio del país (Ciudad de Buenos Aires, Córdoba, Chubut, Mendoza, Neuquén, Río Negro, Santa Fe y Tierra del Fuego) y las que mejoraron su situación (San Juan y Santa Cruz).

Cabe destacar que en un estudio anterior, referido al comportamiento de la mortalidad infantil por jurisdicciones, para la década de los 90 (Mazzeo, 2003), se muestra que, con excepción de San Luis, fueron las mismas jurisdicciones las que se situaron, durante toda la década, por encima del promedio del país. Esto muestra, que si bien el nivel de la mortalidad infantil descendió, se mantuvieron las desigualdades, que se ubicaron en los mismos lugares geográficos.

\subsection{Las políticas y programas de salud y su relación} con la mortalidad infantil

Se considera que el análisis de las políticas y programas, quizás ayude a identificar algunos factores explicativos relacionados con la tendencia de la mortalidad infantil. En Argentina, en el campo de la salud materno-infantil, las estrategias y acciones desarrolladas por el Ministerio de Salud de la Nación, en las últimas dos décadas, buscaron asegurar el derecho de los niños en concordancia con los principios y recomendaciones contenidos en el Artículo 24 de la Convención sobre los Derechos del Niño de 1989. Estas estrategias se implementaron a través de la Dirección de Salud Materno Infantil, instancia responsable del desarrollo y monitoreo de las acciones tendientes al cumplimiento del Plan Nacional de Acción a favor de la Madre y el Niño (Mazzeo, 2003).

Las líneas de acción se desarrollaron a través de programas focalizados: Programa Nacional de Salud MaternoInfantil, Programa Nacional de Alimentación y Nutrición, Programa Nacional de Educación Alimentaria Nutricional, Programa Ampliado de Inmunizaciones, Programas Especiales (de erradicación del sarampión, de la poliomielitis y del tétanos neonatal) y el Programa Materno Infantil y Nutrición (PROMIN). En el 2000 se creó el Programa Nacional de Médicos de Cabecera y la Unidad Coordinadora Ejecutora del Programa de Atención de la Salud Materno Infantil para coordinar las actividades de la Dirección de

Cuadro 8. Categorías de comportamiento de la tasa de mortalidad infantil según división política territorial. Argentina, años 2000-2012.

\begin{tabular}{|l|l|}
\hline \multicolumn{1}{|c|}{ Categoría } & \multicolumn{1}{c|}{ Jurisdicción } \\
\hline $\begin{array}{l}\text { Durante todo el período estuvieron por encima } \\
\text { del promedio del país }\end{array}$ & $\begin{array}{l}\text { Catamarca - Corrientes - Chaco - Formosa - Jujuy - } \\
\text { La Rioja - Misiones - Salta - Tucumán }\end{array}$ \\
\hline $\begin{array}{l}\text { Durante todo el período estuvieron por debajo } \\
\text { del promedio del país }\end{array}$ & $\begin{array}{l}\text { Ciudad de Buenos Aires - Córdoba - Chubut - } \\
\text { Mendoza - Neuquén - Río Negro - Santa Fe - Tierra } \\
\text { del Fuego }\end{array}$ \\
\hline $\begin{array}{l}\text { Comenzaron el período por encima del promedio } \\
\text { del país y luego estuvieron por debajo }\end{array}$ & San Juan - Santa Cruz \\
\hline $\begin{array}{l}\text { Comenzaron el período por debajo del promedio } \\
\text { del país y luego estuvieron por encima }\end{array}$ & Buenos Aires \\
\hline Con altibajos. & $\begin{array}{l}\text { Entre Ríos - La Pampa - San Luis - Santiago del } \\
\text { Estero }\end{array}$ \\
\hline
\end{tabular}

Fuente: elaboración propia en base a MSAS, 2013. 
Maternidad e Infancia con el Promin. Esta unidad cooperó con las provincias mediante transferencias de fondos, según el grado de vulnerabilidad social de cada jurisdicción, para la compra de insumos, capacitación y recursos de asistencia técnica (Mazzeo, 2006).

También se implementó el Programa de Salud Perinatal para disminuir la morbimortalidad materno-infantil, promoviendo en todo el país la salud de las mujeres durante la etapa preconcepcional, el embarazo, el parto y el puerperio y la de sus hijos durante el período fetal y neonatal. Posteriormente, se agregó el Programa Remediar para garantizar el acceso a los medicamentos de quienes están bajo la línea de pobreza o sin cobertura de obra social, prepaga o mutual y que se atienden en los centros de atención primaria de la salud.

En el 2005 surge el Plan Nacer, con el objeto de mejorar la cobertura de salud y la calidad de la atención de las mujeres embarazadas, puérperas y de los menores de 6 años sin obra social. Surge para las regiones del Noreste y Noroeste y en 2007 se extendió a todo el país.

Finalmente, en el 2008, el Consejo Federal de Salud, elaboró un Plan para la Reducción de la Mortalidad Infantil, de la Mujer y Adolescentes. Se trata de focalizar en la modificación de los procesos de gestión, recursos humanos, insumos e infraestructura que resulten un obstáculo para la correcta atención de la población en cada jurisdicción.

Es sabido que las acciones de salud más importantes, por su efecto poblacional, son las preventivas que se brindan a través de la atención primaria. A través del contacto con los servicios de atención pueden detectarse oportunamente riesgos o patologías y revertirlos, así como orientar el comportamiento de las familias hacia conductas de vida más saludables en beneficio directo de los niños (Mazzeo, 2003 y 2006). Al respecto, cabe mencionar que según datos del censo 2010, el 36\% de la población no tenía cobertura en salud y para el grupo menor de 5 años esta proporción se elevaba al 45\%.

La salud de los niños es altamente dependiente de las condiciones de vida en que se desarrolla. La pertenencia a hogares pobres es un factor que puede condicionar tanto las posibilidades de acceso a los servicios como las actitudes en relación con el proceso de salud-enfermedad. Se ha demostrado que la conducta de prevención de la salud infantil por parte de las familias aparece condicionada por la situación de pobreza y por la falta de cobertura médica y que la alta cobertura referida a la consulta durante el primer año de vida está fuertemente asociada a la institucionalización del parto (Mazzeo, 2003). Con referencia a este tema, justamente las jurisdicciones que tienen mayores niveles de mortalidad infantil, son las que registran menores coberturas en salud, llegando en algunos casos incluso a superar el $50 \%$ de falta de cobertura en salud (Catamarca: 39,1\%); Chaco: 57,9\%; Corrientes: 48,4\%; Santiago del Estero: 55,9\%; Formosa:56,9\%; Jujuy: 45,2\%; Misiones:43,6\%; Salta: 47,6\%; San Juan: 44\% y Santiago del Estero: 55,9\%). Asimismo, tiene relación con el porcentaje de parto institucionalizado. Para el total del país se ha incrementado paulatinamente: en 1980 era $89,8 \%$, en 1990 subió a 95,4\%, en 2000 era 97,9\%, llegando a representar en 2012 el 99\% de los nacimientos. Lamentablemente, las jurisdicciones de mayor nivel de mortalidad son también las que presentan menores niveles de parto institucionalizado.

Seguramente, esto se relaciona con los niveles de precarización laboral y de pobreza estructural ${ }^{4}$ que poseen las jurisdicciones. En el total del país, el nivel de hogares con pobreza estructural en 2010 alcanza el 9,13\%, pero es superior en las jurisdicciones con niveles altos de mortalidad infantil (Catamarca: 11,3\%; Corrientes: 15,1\%; Chaco: 18,2\%; Formosa: 19,7\%; Jujuy: 15,5\%; Misiones: 15,6\%; Salta: 19,4\%; San Juan: 10,2\%; Santiago del Estero: 17,6\% y Tucumán: 13,3\%).

Esto demuestra que si bien la amplia mayoría de la población con carencias dirige su demanda de atención a los servicios públicos, parecería que no todos cuentan con este acceso asegurado.

4. Pobreza medida a partir de los hogares con Necesidades Básicas Insatisfechas, es decir hogares que reunieran al menos una de las siguientes características: hacinamiento; vivienda de tipo inconveniente, condiciones sanitarias inconvenientes, niño en edad escolar que no asistiera a la escuela y capacidad de subsistencia. 


\section{Reflexiones finales}

En Argentina aún no se han alcanzado niveles deseables de mortalidad infantil en todo el país Esto lleva a pensar en la insuficiencia de las acciones destinadas a promover y proteger la salud materno-infantil, especialmente en aquellas jurisdicciones que presentan niveles críticos en lo referente a la salud de sus niños. Evidentemente, la creciente desigualdad socioeconómica incidió en las condiciones de salud de los niños de los hogares más pobres, especialmente en aquellos riesgos que pueden ser controlados a través de la estrategia de la atención primaria.

Los recursos financieros constituyen un requisito necesario pero no suficiente, sólo con el respaldo de un compromiso político continuado será posible avanzar hacia la consecución de las metas sociales básicas. La adopción del método de la planificación normativa, a través de la implementación de programas verticales diseñados desde los niveles decisorios centrales, sin la participación de la comunidad, de las autoridades locales y carentes del debido seguimiento y evaluación, revela el carácter tecnocrático de las políticas sectoriales implementadas.

Para reducir las actuales desigualdades, será necesario definir otro tipo de política pública, más inclusiva, que reconozca el carácter médico-social del proceso saludenfermedad y que asegure la protección de los grupos más vulnerables.

\section{Bibliografía}

- Behm, Hugo (1992), Desigualdad social frente a la muerte en América Latina, CELADE, Serie B N 96, Santiago, Chile.

- Breilh, Jaime y Edmundo Granda (1983), Un marco teórico sobre los determinantes de la mortalidad. En Memorias del Congreso Latinoamericano de Población y Desarrollo. Volumen I, UNAM, El Colegio de México, PISPAL, México.

- Bronfman, Mario y Rodolfo Tuirán (1983), La des- igualdad social ante la muerte: clases sociales y mortalidad en la niñez, El Colegio de México, México.

- Calvo, Elvira (1995), Encuesta antropométrica de niños menores de 6 años en INDEC Infancia y condiciones de vida. Encuesta especial para el diagnóstico y la evaluación de metas sociales, Buenos Aires, Argentina.

- Ceballos, María Beatriz (2005), Mortalidad infantil según causas de muerte. Por regiones. República Argentina 1997-2002. VIII Jornadas Argentinas de Estudios de Población, Universidad Nacional del Centro, Tandil, versión CD.

- Celton, Dora y Ribotta, Bruno (2004), Las desigualdades regionales en la mortalidad infantil de Argentina. Niveles y tendencias durante el siglo XX. Presentado al I Congreso de la Asociación Latino-Americana de Población, Caxambú, Brasil.

- CEPAL (2000), Equidad, desarrollo y ciudadanía. CEPAL-Naciones Unidas, Santiago, Chile.

- Cid, Juan Carlos (1995), El peso y la talla de los niños de Salta en la Encuesta de Hogares. En Infancia y Condiciones de vida en INDEC, Infancia y condiciones de vida. Encuesta especial para el diagnóstico y la evaluación de las metas sociales, Buenos Aires, Argentina.

- Delamonica, E., A. Minujin y J. Vandemoortele (2000), Crecimiento, pobreza e infancia. En Socialis. Revista Latinoamericana de Política Social N 3, Ediciones HomoSapiens, Rosario, Santa Fe, Argentina.

- Laurell, Asa Cristina (1986), El estudio social del proceso salud-enfermedad en América Latina, Cuadernos Médicos Sociales No 37, Centro de Estudios Sanitarios y Sociales, Rosario, Santa Fe, Argentina.

- INDEC (1984), La pobreza en la Argentina, Buenos Aires, Argentina.

- López, Elsa y María del Carmen Tamargo (1995), La salud de la mujer. En INDEC, Infancia y condiciones de vida. Encuesta especial para el diagnóstico y la evaluación de las metas sociales, Buenos Aires, Argentina. 
- Mazzeo, Victoria y Raquel Pollero (2005), La mortalidad infantil en ambas márgenes del Río de la Plata en la primera mitad del Siglo XX iDos orillas, dos realidades?, VIII Jornadas Argentinas de Estudios de Población, Universidad Nacional del Centro, Tandil, versión CD.

- Mazzeo, Victoria (2008), Relaciones espaciales entre la situación de la salud-enfermedad de la primera infancia y la desigualdad social en la Ciudad de Buenos Aires entre 1991 y 2002 en Velásquez, Guillermo y Nidia Formiga (Coordinadores) Calidad de vida, diferenciación socio-espacial y condiciones sociodemográficas. Aportes para su estudio en Argentina, Ed. EdiUNS, Bahía Blanca, Argentina, pp. 229-272.

- Mazzeo, Victoria (2007), La mortalidad de la primera infancia en la Ciudad de Buenos Aires en el período 1860-2002. Papeles de población, Año 13, Número 53, julio-setiembre 2007, pp. 241-272.

- Mazzeo, Victoria (2006), La inequidad en la saludenfermedad de la primera infancia. Las políticas de salud y la capacidad resolutiva de los servicios en la Ciudad de Buenos Aires. Tesis de Doctorado. Programa de Doctorado en Ciencia Sociales. FLACSO. www.flacso.org.ar/publicaciones_vermas.php?id $=228$

- Mazzeo, Victoria (2003), La mortalidad infantil en la Argentina iSe cumplirán las metas del año 2000?, IV Jornadas Argentinas de Estudios de Población, Buenos Aires, AEPA.

- Mc Question, Michael J. (2001), Los comportamientos de salud correlacionados y la transición de la mortalidad en América Latina, Notas de Población $\mathrm{N}^{\circ}$ 72, CEPAL, Santiago de Chile.

- Menéndez, Eduardo L. (1992), Grupo doméstico y proceso salud/enfermedad/atención. Del "teoricismo" al movimiento continuo, Cuadernos Médicos Sociales N59, Centro de Estudios Sanitarios y Sociales, Rosario, Santa Fe, Argentina.

- Ministerio de Salud y Acción Social (1999), Metas a favor de la madre y el niño para el año 2000. Avances en el cumplimiento, Buenos Aires, Argentina.

- Ministerio de Salud y Acción Social y Sociedad Argentina de Pediatría (2012), Mortalidad Infantil según Criterios de Reducibilidad, Serie 3, Número 56, Buenos Aires, Argentina.

- Moreno, Elsa (1999), Morbimortalidad en la niñez. En O’Donnell A. y Carmuega E. (coord.) Hoy y mañana. Salud y calidad de vida para la niñez argentina. CESNI Centro de Estudios sobre Nutrición Infantil, Argentina.

- Mosley, W.H. y L.C. Chen (1984), Child survival: strategies for research en Population and Developmente Review Supplement, Vol. 10, Population Council, USA.

- Puffer, R. y C. Serrano (1988), Características del peso al nacer. Publicación Científica N 504, OPS-OMS, Washington, USA.

- Ruzicka, Lado T. (1983), Mortality transition in the Third World: issues for research. Newsletter $\mathrm{N}^{\circ} 17$, IUSSP, Belgique.

- Torrado, Susana (1992), Estructura social de la Argentina 1945-1983. Ediciones de la Flor, Buenos Aires, Argentina.

- Torrado, Susana (1986), Salud-enfermedad en el primer año de vida, Rosario 1981-1982, Informe de Investigación 4, CEUR, Buenos Aires, Argentina.

- Trifiró, María Cristina (2007), La mortalidad infantil en Argentina y Chile: comparación de su evolución desde 1950 y estado actual. IX Jornadas Argentinas de Estudios de Población, Huerta Grande, Córdoba.

- UNICEF-INDEC (2003), Situación de los niños y adolescentes en la Argentina 1990/2001, Serie Análisis Social N², INDEC, Buenos Aires, Argentina.

- Vinocur, Pablo (1999), Exclusión y pobreza. Derechos y oportunidades perdidas de los niños. En O’Donnell A. y Carmuega E. (coord.) Hoy y mañana. Salud y calidad de vida para la niñez argentina. CESNI Centro de Estudios sobre Nutrición Infantil, Argentina. 